\title{
Coastal and island theriofauna of the eastern part of the Gulf of Finland
}

\author{
Andrey G. Bublichenko
}

\begin{abstract}
The history of fauna of the East Coast of the Gulf of Finland and its state under increasing anthropogenic pressure are described in this study. Short characteristics of the current coastal ecosystems of the Gulf of Finland were done. The list of mammal species inhabited the Russian coastal territories of the gulf and adjacent islands is provided. The obtained data demonstrated, that despite of high pressure, which has been brought upon the eastern part of the Gulf of Finland by anthropogenic factor, the main body of the coastal and island mammal fauna, which is inherent for southern taiga forests, has not been lost yet. However, the quota of accidental and rare species is reducing within the major part of the territory. So, the increasing building activity and recreational pressure upon this area call for a close attention to the situation.
\end{abstract}

KEY WORDS: biodiversity, Gulf of Finland, insular isolation, southern taiga species, rare and vulnerable species, anthropogenic impact.

Andrey G. Bublichenko[museum_up@zin.ru and abublichenko@mail.ru], Zoological Institute, Russian Academy of Sciences, Universitetskaya nab., 1, Saint Petersburg 199034, Russia.

\section{Береговая и островная териофауна восточной части Финского залива}

\begin{abstract}
А.Г.Бубличенко
РЕЗЮМЕ. В работе описана история фауны восточного берега Финского залива и ее состояние в связи с увеличивающимся антропогенным воздействием. Даны краткие характеристики современных береговых экосистем Финского залива. Приведен список млекопитающих, населяющих российскую часть побережья Финского залива. Полученные данные свидетельствуют, что, не смотря на высокое давление антропогенного фактора, основная часть береговой и островной териофауны, унаследованной от южнотаежных лесов, не утрачена. Однако, количество редких видов на большей части территории уменьшается. Ввиду увеличивающейся строительной активности и рекреационному давлению необходимо тщательно следить за ситуацией.
\end{abstract}

КЛЮЧЕВЫЕ СЛОВА: биоразнообразие, Финский залив, островная изоляция, южнотаежные виды, редкие и угрожаемые виды, антропогенное давление.

\section{Introduction}

The vertebrate fauna of the north-western part of Russia had not been investigated practically up to fifties of XIX century. The first faunistic generalizations had been done by Yu.I. Simashko and F.I. Brandt in 1850s years (Novikov et al., 1970). The increasing of scientific interest to the local fauna had been outlined since 1890 s and had been connected with the organization of hunting grounds. Later the research papers of V.L. Bianki devoted to mammals inhabited the southern coast of the Gulf of Finland and chiropterans of Saint Petersburg outskirts (Bianki, 1909, 1917) and also other theriological summary reviews (Shnitnikov, 1927; Alekperov, 1938) were published. To the present time several reports about mammals of Leningrad region have been published (Novikov et al., 1970; Danilov et al., 1979; Vereshchagin \& Rusakov, 1979). However, the coastal zone of the Gulf of Finland has been investigated scarcely in spite of availability and the immediate vicinity of Saint-Petersburg. Detailed theriological studies have being begun since merely recent years, therefore it is necessary to obtain more specific data about current species composition and mammals distribution in connection with high rate of economic development within those territories, that is building gas pipelines, ports, cottage settlements, etc. Undoubtedly, specificity of the geographical position of investigated area (especially Karelian Isthmus situated among two large water basins and boarded by the river Neva on south) and the history of landscape formation have affected the local theriofauna. Besides common Palaearctic species, three faunistic elements co-exist there - European, Siberian and, to a lesser degree, Arctic (Novikov et al., 1970; Ivanter, 1986). Palaearctic and arctic species have been remained within the limits of their areal historically, and Siberian species had been forced to move from east to west 
during the glaciation and interglacial periods. Further climate warming and considerable land areas releasing from water gave a possibility to disperse a lignose on these territories (birch, pine, later - oak, lime, elm) and to penetrate into these areas as taiga animal species (from east and north-east) as south species (species of deciduous forests and forest-steppe). Central European species had reached Fennoscandia by two main ways through the southern part of Sweden or by south-eastern way around Littorina Sea (Siivonen, 1976); thereby, the part of Fennoscandia mammal species divided into two races - south-western and south-eastern. Contemporary populations inhabited Karelian Isthmus have an eastern origination. Animals such as Common Squirrel, Hedgehog, Polecat etc. are believed to have appeared in Fennoscandia by "the eastern way", but Flying Squirrel has moved on to west no further than Finland (Hallanaro \& Pylvänäjnen, 2002).

Thus, contemporary mammal fauna of the northwestern part of Russia had been forming for 7000 years due to eastern and south-eastern elements exclusively. Climate had changed approximately for 5000 years ago because of rather low temperature and high humidity, and dark coniferous forests extended widely, broadleaved forests began to contract; so, the species inhabited deciduous forests disappeared or decreased in the number. Typical boreal species appeared following the coniferous forests, and fauna composition became indistinguishable from the modern one.

During the historic times fauna continued to develop under direct or indirect anthropogenic pressure. Development of human settlements triggered not only the changes in landscapes and vegetation but also penetration of sinanthropic mammals. Besides, agricultural activity and forest clearing created favorable conditions for expansion of the open area species - Lepus europaeus, Apodemus agrarius, Micromys minutus, Microtus sgr. arvalis. During the past 200-300 years and especially from the end of XIX - beginning of XX century, several taiga species disappeared as a result of intensive hunting, disturbance's increase, destruction and reduction of habitat places. The abundance of $U r$ sus arctos, Felix lynx, Lutra lutra, Mustela lutreola has been decreased a lot (Airapetyants et al., 1987). For many of the mammal species the northern gulf coast become the border part of their areal (Novikov et al., 1970; Noskov, 2004). It is well known that such populations are vulnerable, have less population density and genetic diversity, as well as sensitive to the slightest changes of habitat conditions. During the historic times such species as Rangifer tarandus silvicola, Alopex lagopus, Gulo gulo and Martes zibellina have disappeared from Karelian Isthmus due to the density reduction in adjacent areas. L. europaeus and Capreolus capreolus have become rarer (Airapetyants et al., 1987). At the same time, border populations often become a basis for species dispersion, the most vivid example of which is Sus scrofa gradually restoring its historical areal in the north-western part of Russia. Thus, areal borders in this region change constantly and therefore, monitoring of local theriofauna composition is necessary.

\section{Materials and methods}

The main goal of our studies conducted in 19942008 was to estimate the modern state of theriofauna of the coastal areas and islands of the eastern part of the Gulf of Finland. For that purpose we have analyzed the mammal fauna composition of the region together with comparative abundance and biotopical distribution of each species, and its status, as well as places and main directions of pathways in this territory. Almost every key territories of south and north coast, from Kurgalsky Peninsula to Vyborgsky Gulf, and the most of islands situated near the coastal line were studied (Fig. 1). Coastal areas were investigated up to the coastal terrace border and beyond, islands and peninsulas - completely. Work arrangement did not provide for estimation of marine mammals current status, but yet some interesting data was obtained.

Conventional methods of captures, winter and summer route accountings, and visual observations were applied in all landscapes including anthropogenic stations, except for the territories of solid building up. The main criteria of comparison of investigated plots included abundance of mammal species, presence or a lack of taiga forms, and a ratio between synanthropic and forest ones. Everywhere we used the same method of trapping and estimation of density in order to obtain comparable data.

Captures were carried out in all kinds of biotopes by different types of traps (snap-traps and Shermann's live-traps) taking into consideration species peculiarities. The traps $(25$ - when we used Sherman's livetraps and 50 - when we used Gero's snap-traps) were put in lines in the range from 10 and $5 \mathrm{~m}$, correspondingly. 2-4 lines of traps, which were used, usually functioned for 3 days. An addition, pitfall traps were dug for shrews capture. Being connected by either "directing fence" or a "channel", those traps were placed in $10 \mathrm{~m}$ between each other. In total, more than 23000 trap-days were conducted during the investigation period, 1490 specimens out of 16 small mammal species were captured.

The obtained data was supplemented by the results of route accountings. The whole route length on the investigated area consisted of more than $6000 \mathrm{~km}, 430$ routes were done, besides the most important routes had been done repeatedly (Tab. 1).

Spatial and biotopic distribution of ungulates and carnivores were estimated on the basis of winter route accounts data (Methods..., 1990; Methodical instruction..., 1997) and summer route accounts, which were carried out by standard methods (Novikov, 1953); for rodents and insectivores - on the basis of captures data obtained according to modified standard methods (Karaseva \& Tselitsina, 1996). All meetings of chi- 


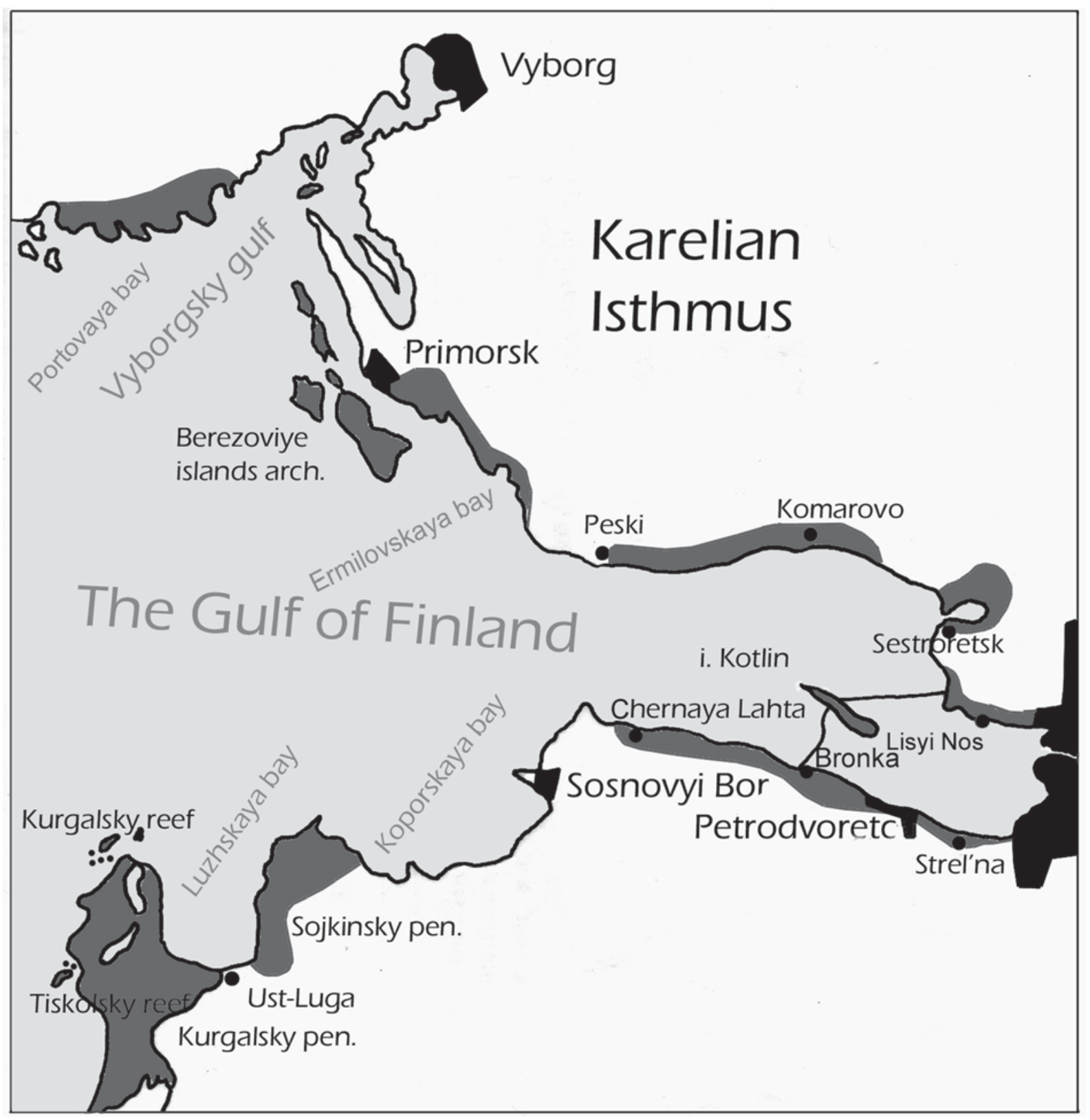

Figure 1. Map of Gulf of Finland with the studied areas shaded in grey.

ropterans during the evening route accounts were fixed; relative estimation of their population density was done according with habitat availability and literature data (Chistyakov, 2002a, b).

The mapping of routes and the sites of rare species location was conducted on the topographical map $\mathrm{M}$ 1:500 000 moreover GPS Etrex Garmin Legend has been used since 2004.

The list of mammal species inhabited the key areas of coastal territories of the eastern part of the Gulf of Finland and adjacent islands was made on a base of obtained data, the status of permanent residence and degree of vulnerability are marked for every species (Tab. 3). The main criterion for the determination of the species state within the area was the frequency of marks of its vital activity on the route, and for rodents and insectivore - the results of trapping.

The results are presented by use of conventional scale of mammal abundance (Tab. 2), which gives an opportunity not smoothing over the basic tendencies in ratio of population density to grade its perennial variability and, thus, get rid of needless data.

The results of our examination were being compared with the published data about abundance of concrete mammal species within slightly damaged forest areas of the Leningrad Region. The information about game species was also compared with the data of censuring, which have been got by game inspection. The 
Table 1. The main characteristics of permanent monitoring route accounts on the key territories.

\begin{tabular}{|l|l|l|l|l|}
\hline Locality & $\begin{array}{l}\text { Number of } \\
\text { permanent routes }\end{array}$ & $\begin{array}{l}\text { Duration of } \\
\text { survey }\end{array}$ & $\begin{array}{l}\text { Total extension of } \\
\text { routes (in km) }\end{array}$ & Season of observations \\
\hline Kurgalsky Peninsula & 13 & 8 & 1900 & during the whole year round \\
\hline Sojkinsky Peninsula & 9 & 1 & 140 & spring-summer \\
\hline Chernaya Lahta -Bronka & 4 & 4 & 320 & summer-autumn \\
\hline Petrodvorets - Strel'na & 6 & 3 & 210 & during the whole year round \\
\hline Lisyi Nos -Sestroretsk & 7 & 3 & 400 & during the whole year round \\
\hline Komarovo - Chernaya & 9 & 3 & 380 & summer-winter \\
\hline Primorsk -Ermilovskaya & 6 & 2 & 340 & summer-autumn \\
\hline Portovaya Bay & 11 & 5 & 900 & during the whole year round \\
\hline Kotlin Island & 3 & 2 & 120 & spring-summer \\
\hline Berezovye Archipelago* & 19 & 5 & 1100 & spring-autumn \\
\hline Small Islands & 34 & $1-2$ & 300 & summer \\
\hline
\end{tabular}

* The data, which concern the largest islands of the archipelago.

Table 2. The conventional scale of mammal abundance applied to itinerary examination.

\begin{tabular}{|l|l|l|}
\hline Denomination & Extent of abundance & Content \\
\hline a & Abundant & $\begin{array}{l}\text { The species, which occurs in considerable abundance during each of } \\
\text { diurnal/vespertine examination (the concrete rates of population density } \\
\text { depend on current conditions of the season and year) }\end{array}$ \\
\hline $\mathrm{c}$ & Common & $\begin{array}{l}\text { The species, which is practically traceable or trappable during each of } \\
\text { diurnal/vespertine examination (the concrete rates of population density } \\
\text { depend on current conditions of the season and year) }\end{array}$ \\
\hline $\mathrm{r}$ & Rare & $\begin{array}{l}\text { The species, whose frequency of occurrence is no higher, than 1-2 times within } \\
\text { the total period of survey. }\end{array}$ \\
\hline $\mathrm{s}$ & Sporadic & $\begin{array}{l}\text { The species, which occurs (or can be trapped) 1-2 times within the season of } \\
\text { survey, but not annually. }\end{array}$ \\
\hline$?$ & Indefinite & $\begin{array}{l}\text { The existence of the species within the territory of survey is possible judging } \\
\text { by physiographic factors, the data from literature and results of questioning. }\end{array}$ \\
\hline
\end{tabular}

The average extension of diurnal/vespertine route was $10-15 \mathrm{~km}$. Duration of itinerary survey was 9 h. The detection of fresh marks of mammal's vital activity was also considered as the occurrence.

preliminary evaluation of availability of each control area for a permanent presence of a particular mammal species was being carried out. It included the food capacity and safety of stations, the size ratio between continuous woodland areas and optimal habitats for each group of species (Martynov, 1979).

In total, 51 species were registered on the coastal territories, islands and adjoining water area during the entire research period. Among them, 10 species are rare in Leningrad region, 11 species are included into the Red Data Book of the Baltic Region (1993) and into the Red Data Book of East Fennoscandia (1998), 2 species - into the Red Data Book of Russian Federation (2001) and 2 species - into the Red Data Book of IUSN (2007).

\section{Results}

Detailed observations were conducted in 1994-2005 on the protected natural territory Kurgalsky Peninsula (Fig. 1), where the theriofauna has the richest diversity of the investigated coastal area (50 out of 51 species detected on the whole territory; see Tab. 3). Erinaceus europaeus, Sylvimus flavicollis, Eliomus quercinus and other species of southern fauna appeared there due to nemoral floristic complexes existing. For instance, the population of orchard dormouse, which included no more than 20-30 individuals, was registered in the northwestern part of the peninsula only. Castor fiber, Lutra lutra, Mustela lutreola and M. vison were marked on Beloe Lake and adjacent streams. The presence of the European mink, which inhabited the Peninsula in the beginning of 1990-ths, as the species has not been trapped since that time (Pchelintsev, personal communication). The population density of Cervus nippon and Cervus elaphus, which were introduced there in 1977, is also being reduced; the groups of 3-7 animals still occur in the central and north-eastern parts of the peninsula. Sus scrofa occurs all over the territory of Peninsula, preferring a reed tangle on the north-eastern and northern parts of the peninsula during the summer period. Three bear's dens were found out on the southeastern part of the peninsula, in the same place the couple of Felix lynx is regularly registered. The comparative abundance of rodents, insectivores and carnivores in their all typical biotops is close to that in the 


\begin{tabular}{|c|c|c|c|c|c|c|c|c|c|c|c|c|c|c|c|c|c|c|c|c|c|c|c|c|c|c|c|c|}
\hline 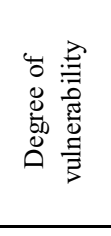 & & I & & & & & $\tilde{\widetilde{x}}$ & 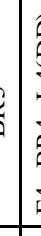 & 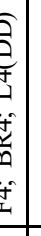 & 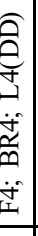 & 在 & 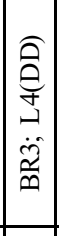 & & $\stackrel{\tilde{\mu}}{\tilde{m}}$ & 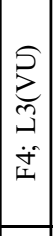 & & & & 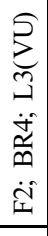 & & & & & & & & & \\
\hline 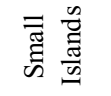 & 1 & 1 & \pm & 1 & 1 & 1 & 1 & & I & 1 & 1 & 1 & \pm & 1 & 1 & 1 & 1 & I & 1 & 1 & 1 & 1 & 1 & I & 1 & I & \pm & \pm \\
\hline 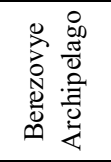 & 1 & \pm & \pm & \pm & ts & \pm & - & & - & 1 & $\infty$ & $\infty$ & $t$ & 1 & 1 & \pm & 1 & 1 & 1 & 1 & 1 & \pm & 1 & 1 & \pm & 1 & \pm & J \\
\hline 可咅 & 1 & is & 1 & 1 & । & 1 & $t$ & 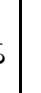 & -1 & 1 & \pm & 1 & in & 1 & 1 & 1 & 1 & 1 & 1 & 1 & 1 & 1 & 1 & \pm & $\underset{+}{+}$ & 1 & 1 & \pm \\
\hline 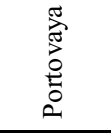 & $t$ & \pm & $\underset{\tau}{t}$ & \pm & \pm & \pm & 0 & & -1 & 1 & 1 & 1 & $\stackrel{+}{+}$ & $\infty$ & 声 & \pm & \pm & \pm & 1 & 1 & \pm & 1 & \pm & 1 & \pm & 1 & 1 & $\underset{t}{t}$ \\
\hline 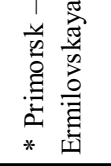 & \pm & 1 & む & 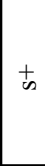 & I & 1 & 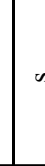 & s & $\infty$ & 1 & 1 & 1 & \pm & 1 & 1 & \pm & 1 & 1 & 1 & 1 & \pm & \pm & 1 & 1 & \pm & 1 & 1 & J \\
\hline 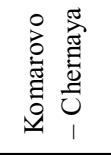 & $\begin{array}{l}t \\
0\end{array}$ & \pm & $\underset{\tau}{t}$ & \pm & \pm & \pm & $r$ & 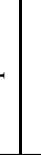 & I & 1 & $\infty$ & 1 & $\stackrel{+}{0}$ & 1 & \pm & \pm & \pm & 1 & 1 & \pm & \pm & \pm & \pm & \pm & t & 1 & 1 & J \\
\hline 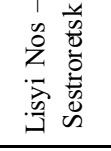 & t) & \pm & $\begin{array}{l}+ \\
\end{array}$ & \pm & I & \pm & 1 & & - & 1 & - & 1 & \pm & 1 & 1 & $\begin{array}{l}+ \\
0\end{array}$ & 1 & \pm & 1 & 1 & $\underset{t}{t}$ & \pm & 1 & \pm & \pm & \pm & $\stackrel{t}{t}$ & $\underset{\Phi}{+}$ \\
\hline 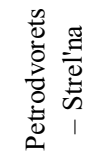 & t & \pm & $\begin{array}{l}+ \\
0\end{array}$ & \pm & \pm & \pm & $t$ & $=1$ & - & - & \pm & $\infty$ & \pm & \pm & 1 & $\begin{array}{l}+ \\
0\end{array}$ & 1 & 1 & 1 & \pm & $\begin{array}{l}t \\
t\end{array}$ & 1 & さ & $\begin{array}{l}t \\
0\end{array}$ & $\underset{\omega}{t}$ & $\stackrel{ \pm}{=}$ & \pm & $\begin{array}{l}+ \\
\end{array}$ \\
\hline 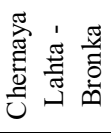 & t & \pm & $\stackrel{t}{ \pm}$ & \pm & I & \pm & \pm & $E$ & \pm & \pm & \pm & 1 & $\begin{array}{l}+ \\
0\end{array}$ & \pm & 1 & $\begin{array}{l} \pm \\
0\end{array}$ & 1 & \pm & 1 & \pm & $\stackrel{+}{\star}$ & 1 & \pm & \begin{tabular}{|l}
$t$ \\
\end{tabular} & $\stackrel{t}{t}$ & 1 & \pm & t \\
\hline 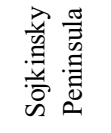 & $\stackrel{+}{t}$ & \pm & $\stackrel{+}{t}$ & \pm & \pm & $\stackrel{+}{0}$ & + & t & - & -1 & \pm & $\infty$ & $\underset{\sigma}{t}$ & 1 & 1 & $\begin{array}{l}+ \\
0\end{array}$ & 1 & $\begin{array}{l} \pm \\
0\end{array}$ & 1 & \pm & \pm & \pm & $\stackrel{+}{=}$ & 1 & I & 1 & ' & $\underset{\Phi}{ \pm}$ \\
\hline 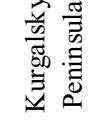 & t) & 声 & $\stackrel{+}{t}$ & ${ }_{0}^{+}$ & \pm & $\stackrel{+}{\circlearrowright}$ & + & t. & - & -1 & \begin{tabular}{|l}
$t$ \\
0
\end{tabular} & $\infty$ & $\underset{\sigma}{ \pm}$ & \pm & \pm & $\stackrel{+}{+}$ & 1 & む & 南 & \pm & む & \pm & $\stackrel{t}{t}$ & $\begin{array}{l}t \\
\end{array}$ & $\stackrel{+}{ \pm}$ & \pm & \pm & $\underset{+}{+}$ \\
\hline $\begin{array}{l}\frac{\infty}{0} \\
\frac{0}{0} \\
\frac{n}{n}\end{array}$ & 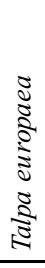 & 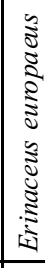 & 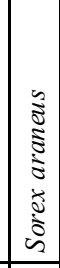 & 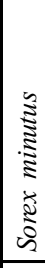 & 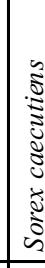 & 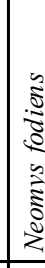 & a & 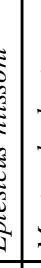 & 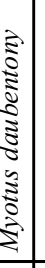 & 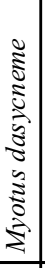 & 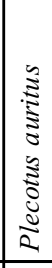 & 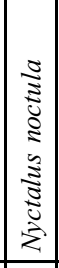 & 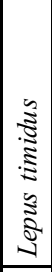 & $\mid$ & 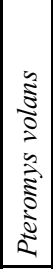 & 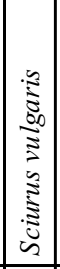 & 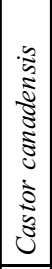 & 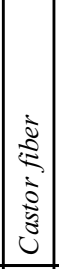 & 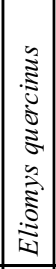 & 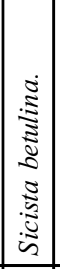 & 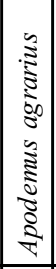 & 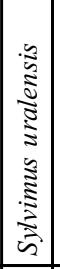 & 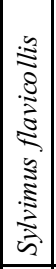 & 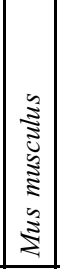 & 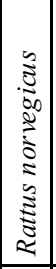 & 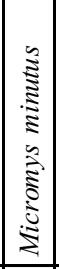 & 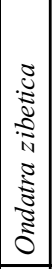 & 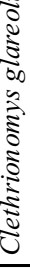 \\
\hline$\dot{z}$ & - & $\mathrm{N}$ & $\mathrm{m}$ & $\theta$ & in & 6 & & & $\infty$ & $a$ & 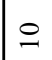 & $=$ & $\simeq$ & $\cong$ & \pm & $\because$ & 0 & $=$ & $\stackrel{\infty}{-1}$ & 2 & $\stackrel{i}{i}$ & $\vec{\sim}$ & ה & $\tilde{\imath}$ & $\stackrel{d}{\sim}$ & $\approx$ & $\stackrel{\sim}{\sim}$ & $\hat{\sim}$ \\
\hline
\end{tabular}




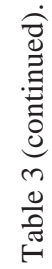

\begin{tabular}{|c|c|c|c|c|c|c|c|c|c|c|c|c|c|c|c|c|c|c|c|c|c|c|c|c|c|}
\hline 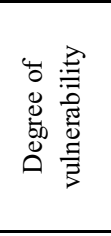 & & & & & & (ุ) & & & & & & & 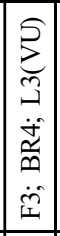 & & & & $m$ & & & & 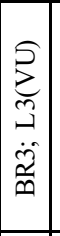 & & & 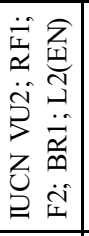 & 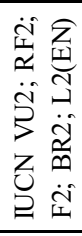 \\
\hline 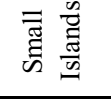 & 1 & \pm & I & 1 & 1 & 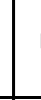 & & $\begin{array}{l}+ \\
0\end{array}$ & \pm & 1 & 1 & 1 & I & $\infty$ & I & 1 & 1 & 1 & 1 & 1 & 1 & 1 & 1 & 1 & $\infty$ \\
\hline 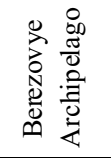 & 1 & \pm & 1 & 1 & $\infty$ & 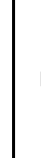 & & $\begin{array}{l} \pm \\
0\end{array}$ & \pm & 1 & \pm & 1 & 1 & $\stackrel{+}{0}$ & 1 & \pm & 1 & 1 & \pm & \pm & 1 & 1 & 1 & r. & \pm \\
\hline 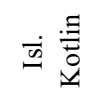 & 1 & \pm & 1 & 1 & 1 & . & & \pm & 1 & 1 & 1 & 1 & 1 & $n$ & 1 & 1 & 1 & 1 & 1 & 1 & 1 & 1 & 1 & 1 & 1 \\
\hline 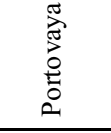 & 1 & \pm & \pm & \pm & $\stackrel{+}{0}$ & 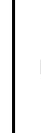 & & \pm & $\begin{array}{c}t \\
\end{array}$ & \pm & \pm & 1 & 1 & $\begin{array}{l} \pm \\
0\end{array}$ & $t$ & \pm & $\infty$ & \pm & ${ }_{0}^{+}$ & \pm & 1 & 1 & 1 & 1 & 1 \\
\hline 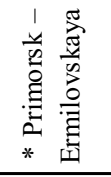 & 1 & t & 1 & 1 & 1 & 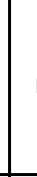 & & 1 & \pm & 1 & \pm & 1 & 1 & \pm & 1 & $\infty$ & 1 & 1 & 1 & 1 & 1 & 1 & 1 & 1 & 1 \\
\hline 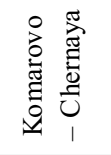 & 1 & t & t & \pm & \pm & . & & $\begin{array}{c}+ \\
0\end{array}$ & \pm & \pm & \pm & \pm & $\infty$ & $\stackrel{t}{0}$ & 1 & $\infty$ & 1 & 1 & \pm & \pm & 1 & 1 & 1 & 1 & 1 \\
\hline 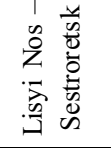 & 1 & \pm & \pm & t & 1 & 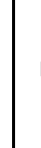 & & $\begin{array}{c} \pm \\
0\end{array}$ & \pm & \pm & $\stackrel{ \pm}{ \pm}$ & 1 & 1 & $\stackrel{+}{0}$ & 1 & 1 & 1 & 1 & $\infty$ & $\infty$ & 1 & 1 & 1 & 1 & $\infty$ \\
\hline 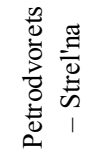 & 1 & ${ }_{\infty}^{+}$ & \pm & \pm & + & 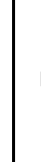 & & \pm & + & \pm & \pm & 1 & 1 & \pm & 1 & $n$ & 1 & 1 & $\infty$ & $\infty$ & 1 & 1 & 1 & 1 & 1 \\
\hline 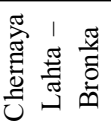 & । & t & \pm & \pm & \pm & 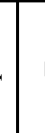 & & \pm & \pm & \pm & \pm & 1 & 1 & t & 1 & 1 & 1 & $\begin{array}{l}t \\
\omega\end{array}$ & \pm & \pm & $\begin{array}{l}t \\
\omega\end{array}$ & 1 & 1 & 1 & 1 \\
\hline 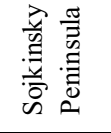 & 1 & む & \pm & $t$ & \pm & 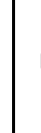 & & $\begin{array}{c} \pm \\
\text { t }\end{array}$ & $\begin{array}{l} \pm \\
0\end{array}$ & t & $\stackrel{t}{0}$ & \pm & 1 & $\begin{array}{l} \pm \\
0\end{array}$ & - & $\begin{array}{l} \pm \\
0\end{array}$ & 1 & 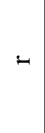 & $t$ & $\begin{array}{l} \pm \\
0\end{array}$ & -4 & 1 & 1 & 1 & $n$ \\
\hline 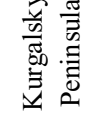 & \pm & t & t & \pm & 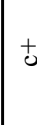 & o & . & $\begin{array}{c}t \\
0\end{array}$ & \pm & t & t & \pm & \pm & $\underset{\omega}{+}$ & $t$ & $\begin{array}{c} \pm \\
0\end{array}$ & \pm & \pm & $t$ & t & \pm & t & t & $H$ & \pm \\
\hline 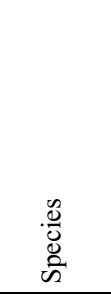 & 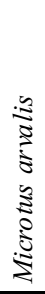 & 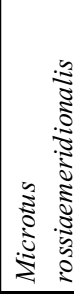 & 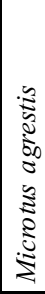 & 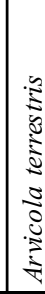 & 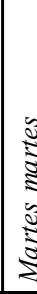 & (3) & 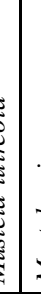 & 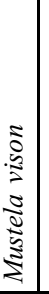 & 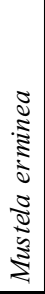 & 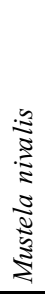 & 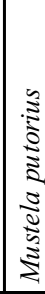 & 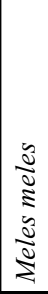 & 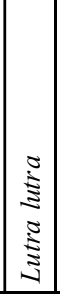 & 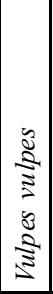 & 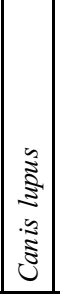 & 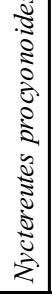 & 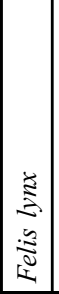 & 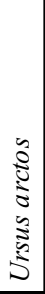 & 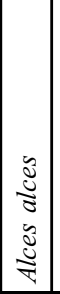 & $\begin{array}{c}0 \\
0 \\
0 \\
5 \\
5 \\
5 \\
5\end{array}$ & 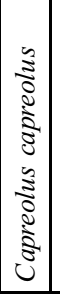 & 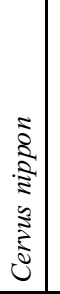 & 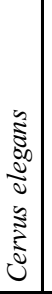 & 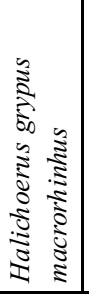 & 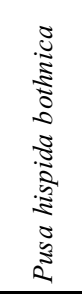 \\
\hline$\dot{z}$ & $\stackrel{\infty}{\sim}$ & iे & in & $\bar{m}$ & ल & & ر & 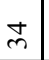 & $m$ & $\stackrel{0}{m}$ & $\bar{m}$ & $\stackrel{\infty}{m}$ & mे & \& & $F$ & f & $f$ & F & if & $\stackrel{0}{+}$ & f & $\stackrel{\infty}{+}$ & qे & in & $\bar{n}$ \\
\hline
\end{tabular}


intact woodlands of other regions of the North-East of Russia. Kurgolovskaya Reyma and Tiskolsky Reef islands situated near Kurgalsky Peninsula are the places of seasonal herds of Pusa hispida bothnica; Halichoerus grypus prefers distant islands (Hiitamatala, Moshny and others).

Investigations on Sojkinsky Peninsula (Fig. 1) were conducted in 1998. In total, 35 mammal species have been found out there, within that territory many taiga species were quite common or even abundant (Tab. 3). Mammal fauna is exceptionally rich in the forest areas (mixed coniferous-broad-leaved and deciduous forests mostly) adjacent to forest streams and rivers - Belaya, Chernaya, Khabolovka. In addition to typical sylvatic species, such aquatic and semi-aquatic ones as Neomys fodiens, Myotis spp., Castor fiber, Arvicola terrestris, Mustela vison, Nyctereutes procyonoides are common there (Tab. 3). Talpa europaea, some shrew species, Clethrionomys and Sylvimus species, Mustela erminea and $M$. nivalis are widespread in old mixed forests in the coastal terraces and in the south-eastern part of the peninsula. Two settlements of Meles meles exist there too. In biotopes of all kinds Lepus timidus, Sus scrofa, Mustela putorius and Vulpes vulpes were registered. Canis lupus and Ursus arctos are not so common, being rare in many patches.

The territories from Bronka village to Chernaya Lahta village (Fig. 1) were investigated in 1997-2001. In total, 34 mammal species were registered there; however, the population density of taiga mammal species, particularly large carnivores and ungulates is rather law at this coastal site because of high anthropogenic pressure (Tab. 3). Only Talpa europaea, Sorex araneus, Sciurus vulgaris, Clethryonomys glareolus and Microtus spp. are common in the observed coastal area; Arvicola terrestris, Ondatra zibetica and Neomys fodiens were registered from time to time in their accepted biotops. Beyond the coastal terrace border, where large forest areas have remained within Lomonosov city and Bolshaya Izora village vicinities, the number of mammal species is getting higher - Alces alces, Sus scrofa, Capreolus capreolus, Ursus arctos and several species of small mustelids have been registered there, while Castor fiber and Mustela vison occur in the rivers and streams. However, none of those species can be considered as abundant or common.

The analogous situation is observed in old abandoned parks of palace complexes, where in spite of formally high level of mammal diversity, (34 species

\footnotetext{
Notes to Table 3 :

The status of permanent residence: a - abundant; c - common; $\mathrm{r}$ - rare; $\mathrm{s}$ - sporadic; ? — indefinite; + — breeds; - — absent Degree of vulnerability: IUCN Red Book. VU2 — vulnerable Red Data Book of Leningrad region. L2(EN) - endangered; L3(VU) - vulnerable; L4(DD) — data deficient

Red Data Book of Baltic Region. BR2 - vulnerable; BR3 - least concern; BR4 - declining

Red Data Book of East Fennoscandia. F2 — vulnerable; F3 — rare; F4 - declining

* _ by the 2004
}

are existing at the present time), the hoofed mammals and most of carnivores are rare and occasional (Bakhmatova et al., 2005).

The coastal zones from Petrodvorets to Strelna (Fig. 1) were investigated in 2001-2002 and have the least number of species (12), which were registered on the coastal building area (Tab. 3). Talpa europaea, Sorex araneus, Microtus rossiaemeridionalis, Apodemus agrarius, Mustela putorius, from time to time $-M$. nivalis were observed along the wet black alder forest border, single tracks of Lepus timidus and Vulpes vulpes were marked there too.

The fauna composition of the northern coastal areas of the gulf lying within Lisiy Nos and Sestrotetsk vicinities (Fig. 1) were observed in 2005-2007, includes 28 species of terrestrial mammals (Tab. 3). In addition to that, the solitary herd of Pusa hispida bothnica was marked in adjoining water area. The mire massif near artificial lake Sestroretsky Razliv has the least number of species (6). Among semi-aquatic forms Neomys fodiens and Castor fiber are to be found within Razliv Lake and the Sestra and Chernaya rivers, but the most abundant (up to 400 occurrences per $13 \mathrm{~km}$ of the coastline) was Ondatra zibethica. Sus scrofa inhabits reed tangle, coastal meadows and forest margins. Maximum diversity and high population density were registered in a bush growing along the border of shore sands. The most common species were Apodemus agrarius, Sylvimus uralensis, Clethrionomus glareolus, Sorex araneus, there were Vulpes vulpes, Mustela nivalis, $M$. putorius from carnivores, and Myotus spp. were observed occasionally above the streams and channels.

The fauna of the coastal zone within Komarovo village and the river Chernaya mouth areas to the point of Peski village (Fig. 1) investigated in 2001, 20052006 can vary from 20 species in densely built-up Komarovo vicinities (Baranova et al., 2002) to 32 species (coastal patch around the rivers Gladyshevka and Chernaya mouthes). Sorex araneus, Clethrionomys glareolus, Sciurus vulgaris, Lepus timidus, Vulpes vulpes as species, which are the most resistant to anthropogenic pressure, are rather common in the most of the examined territory. The main negative factors for large taiga species existing within the region of the coastal building up are the land development and changing in the natural ecosystems as a result. The significance of the coastal terraces, sand dunes and adjoining water areas as constant habitats is not so big. The forest types diversity (coniferous, pine, mixed forests) on the rivers Gladyshevka and Chernaya and the nearest coastal areas in spite of high anthropogenic pressure creates various conditions for different mammal species existing: Lutra lutra, Neomys fodiens, Mustela vison inhabit the flood lands, Sorex minutus, Microtus agrestis, Mustela erminea and Martes martes are common in coniferous forests, some chiropterans, Eptesicus nilssoni first of all, occur in coniferous-mixed forests there, as well as Meles meles, Alces alces, which are less abundant. However, in spite of comparatively high level of 
species diversity, the population density of most of taiga species within that territory is not high (Tab. 3).

A close attention was attended to the coastal zones from Ermilovskaya bay to Primorsk port (Fig. 1). At the beginning of our work in 1996 mammal fauna had included 23 species only because of long-term human activities within that territory (Bublichenko et al., 2003). Monitoring investigations were conducted in 2002 2004, when Primorsk port building had been finished and data analysis showed the decreasing of mammal diversity at the expense of large carnivores and ungulates uppermost - Canis lupus, Alces alces, Sus scrofa. Other of mammal species - Eptesicus nilssoni, Sylvimus uralensis, Sciurus vulgaris, Mustela erminea, $M$. vison and Nyctereides procynoides were being registered occasionally. The stable, but not high is population density of Lepus timidus, Mustela putorius, Vulpes vulpes maintained, Microtus rossiaemeridionalis and Sorex araneus were the only 2 species, whose population density has been considerably increased in several plots (Tab. 3).

The western coast of Vyborgsky Bay (Fig. 1) including Portovaya bay was observed in 2003-2008. In spite of the law degree of vegetation diversity and longterm anthropogenic landscape changing 33 species inhabit in several old-growth forest areas, where the species composition of rodents, insectivores and carnivores is typical for south-taiga zone. The dominant species were Sorex araneus, Clethrionomys glareolus and Sciurus vulgaris; S. minutus, Sylvimus flavicollis and Microtus agrestis were not so common there (Tab. 3). Mustela erminea and M. putorius have the most considerable abundance among small mustelids; the density population of $M$. nivalis is rather law. Canis lupus at least three couples, and many occurrences of Vulpes vulpes were registered within all investigated area. Ursus arctos and abundant Martes martes were registered in old-growth coniferous forests. Ungulates were presented by two species - Alces alces and Sus scrofa less abundant. Well-known periodical invasions of elks from the border regions of Finland across Karelian Isthmus described in literature (e.g., Köppen, 1883; Timofeeva, 1974) led to the creation of local zones with high animal concentration along the western border of the investigated area. Elks are forced to move along the frontier barbed wire entanglement not having a possibility to disperse or change a direction. It is quite possible that some animals pass the obstacles through water or ice of the gulf; we registered numerous elk tracks on the coastal meadows there quite often.

In spite of reduction of comparative population density of secondary and rare sylvatic mammal species the species composition of the local theriofauna has been typical for south taiga, exploited for many years. However, numerous trees felling in the large forest massifs in 2005-2007 had decreased biodiversity very much, we registered the isolated holes of Microtus rossiaemeridionalis and $\mathrm{Cl}$. glareolus only in such places.
The faunistic observations on the Kotlin Island territory (Fig. 1) were carried out in 2004 and 2007. The peculiarities of vegetation and extremely high degree of anthropogenic pressure equally with insular isolation determined the scanty mammal fauna composition -11 species (Tab. 3), including bats who spend the winter in the forts of Kotlin (Eptesicus nilssoni and Plecotus auritus mostly) (Chistyakov, 2002a), and two carnivores species - Mustela vison and Vulpes vulpes. In general, mammals are distributed in the western coast of the island, along the coastline and in the wet mixed and small-leaved forests.

One of the most interesting investigated areas is "The Berezoviye Islands" regional complex sanctuary which includes more than 30 variously sized islands (Fig. 1). Detailed observations were conducted there in 2000-2005. The archipelago mammal fauna is not so representative (24 terrestrial species and 1 marine species - Pusa hispida bothnica, and it possible, that Halichoerus grypus macrorhinhus appears in this part of water area occasionally), that it is connected with limited dispersion possibilities and the conditions of insular isolation. The fauna of the largest island Bolshoy Berezoviy includes 20 species, 14 species are to be found on Severny Berezoviy Island, 16 species - on Zapadny Berezoviy Island, 5 species - on Maly Berezoviy Island. The number of species in small islands varies from 1 to $4-5$. The permanent background species of the islands are Sorex araneus, Clethrionomys glareolus, Lepus timidus, Mustela vison and Vulpes vulpes. Sciurus vulgaris, Mustela erminea, M. putorius, Ondatra zibethica and Nyctereutes procyonoides are to be found in the largest islands in preference, everywhere having not very high population density (Tab. 3). The archipelago mammal's diversity can vary noticeably from year to year. Thus, Alces alces and Sus scrofa had been absent in 2002-2003 on the investigated area, but in 2004-2005 five-seven mooses and more than a dozen of wild boars dispersed through the largest islands. Canis lupus appears in winter from time to time on Bolshoy Berezoviy Island. Several species such as Meles meles and Ursus arctos which are spread in the mainland coastal areas never occurred in the archipelago, though the biotops are available for them. Probably, the terrestrial mammals having the winter sleep can not overcome the Bjerkesund strait $2 \mathrm{~km}$ in width. It is necessary to take into account that the reasons of several species absence (Pteromys volans and several small rodent species) can be as species peculiarities as coincidence. In regard to the islands fauna of the Vyborgsky Gulf observed at the same time, species composition was similar to small islands of the Berezoviye Islands archipelago.

\section{Discussion}

Mammal habitat is ambiguous in the area investigated. Even excluding the regions of immediate urban construction, fauna composition is similarly poor in the 
city outskirts (from Lahta to Peski village on the north coast and from Strel'na to Chernaya Lahta on the southern coast (Bublichenko \& Bublichenko, 2005a). It's driven by the composition of tree species and absence of extensive undisturbed forestland. Besides, the leading role is being played by prolonged anthropogenic changes of the landscapes, presence of railroads and coastal highways, intensive building along the coast, pine-forest cutting on the upper terrace, and pressure from increasing recreational activities. Such regime creates constant disturbance to large mammals; only eurybionts are able to exist there constantly. Typically, we registered 12-15 mammal species at most on such territories, though in other conditions fauna diversity could be more extensive even in urban areas. The later is characteristic feature of the protected nature areas and coastal abandoned parks which have been previously included into palace complexes of the southern coast of the Gulf of Finland (Bakhmatova et al., 2005). Old parks have some attractive features for many species: large size of territory, trees species diversity of and high number of deciduous ones. They attract the representatives of the southern taiga fauna (Sorex araneus, Clethrionomys glareolus, Sciurus vulgaris, and Lepus timidus) and inhabitants of nemoral forests (Erinaceus europaeus, Sylvimus flavicollis) which can coexist there. The rich nutritive base and favorable environmental conditions in the unrestored park areas also attract the small predators - Mustela putorius, Mustela erminea, M. vison, M. nivalis, Martes martes and Vulpes vulpes were registered here. The most interesting sites of the urban zone of the northern gulf coast are Sestroretsky Razliv and Gladyshevka river valley, near Peski village (Bublichenko \& Bublichenko, 2005b). Despite of increased recreational pressure during the summer-autumn period, mammal fauna composition is still diverse, and dominant species abundance can reach high indexes. Neomys fodiens, Castor fiber, Mustela nivalis, M. vison, and Meles meles were registered there routinely. Alces alces, Sus scrofa and Nyctereides procynoides observed there in the past decades have become much rare.

Despite of the presence of large settlements along the entire coastal line (Primorsk, Vyborg, Sosnovyj Bor, Ust-Luga), coastal ecosystems of the west part of the gulf as yet have not been impacted by high anthropogenic pressure to the degree of the eastern areas. The main reason for that is state border regime limiting constructional, recreational and other human activities. This situation allowed preservation of unique nature complexes of Kurgalsky Peninsula where rare mammals of the region still exist (Pteromys volans, Eliomys quercinus, Lutra lutra, Felix lynx) (Volkova et al., 2001). Herds of Halichoerus grypus and Pusa hispida bothnica were observed on the banks of Tiskolsky and Kurgalsky reefs; these are the only herd of Halichoerus grypus registered on the Russian offshore of the Gulf of Finland. The worse situation is in the region the Gulf of Vyborg, where, in spite of a large total areas of wood- land, active building up and timber cutting have already led to abnormality of theriofauna's structure and decrease of density of secondary and rare forest species.

The island fauna turned out to be relatively poor, primarily due to the restricted opportunity for group dispersion and insular isolation. The maximum number of species detected was 24 (Berezoviye Islands archipelago) (Bublichenko 2006, 2007), for other islands it varied from 0 (small rocky islands) to 8-14 species. The recreation and building up pressure have not much influenced those territories, but now they are getting more and more significant.

The data obtained demonstrated that despite of high anthropogenic pressure the coastal and island mammal fauna of the eastern part of the Gulf of Finland continue to maintain its typical species composition inherent for southern taiga forests. Nevertheless, increased building activity and recreational pressure in this area, including the zone of existing protected areas, call for a close attention to the situation and development of viable measures to protect key biodiversity areas in this region.

ACKNOWLEDGMENTS. This study was supported by the Saint-Petersburg Research Centre of Russian Academy of Sciences, Biodiversity program of RAS and KE-Association (Russia). I express sincere gratitude for all my expedition colleagues, particularly to J. Bublichenko, A. Tikhonov, A. Abramov, and K. Tretyakov.

\section{References}

Alekperov A.M. 1938. [Terrestrial Vertebrate Fauna of Southern Taiga Subzone of Leningrad Region]. Leningrad. 26 pp. [in Russian]

Airapetyants A.E., Strelkov P.P. \& Fokin I.M. 1987. [Nature of Leningrad Region. Mammals]. Leningrad: Lenizdat. 143 pp. [in Russian]

Bakhmatova K.E., Vasilyeva V.A., Vershinina O.M., Vlasov D.Yu., Gimelbrandt D.E., Ivanov A.A., Matinjan N.N., Osipov D.V., Pchelintsev V.G., Rumyantseva E.E., Ryabova V.N., Ukhacheva V.N., Cherepanova N.P., Chistjakov D.V. \& Chmilevskky D.A. 2005. [Sergievka Park - a complex nature monument]. Pavlovsk-SanktPeterburg: Pavel. 143 pp. [in Russian, with English summary]

Baranova E.V., Baranov V.P., Bibikova T.V., Bublichenko A.G., Bublichenko J.N., Volkova E.A., Isachenko G.A., Kataeva O.A., Kurbatova L.E., Potapov R.L. \& Khramtsov V.N. 2002. [Komarovoi coast - a complex nature monument]. Sankt-Peterburg: Boston-Spektr. 91 pp. [in Russian, with English summary]

Bianki V.L. 1909. [Notes on mammals inhabited coastal line of Petergof District between villages Lebyazh'e and Chernaya Lahta] // Ezhegodnik Zoologicheskogo Muzeya Akademii Nauk. T.14. No.1-2. P.107-118 [in Russian].

Bianki V.L. 1917. [Our current knowledge about Chiroptera of Petrograd region] // Ezhegodnik Zoologicheskogo Muzeya Akademii Nauk. T.21. No.1--2. P. 33-36 [in Russian]. 
Bublichenko A.G. 2006. The population status of game mammals in the Berezoviye Islands archipelago, the Gulf of Finland // Dynamics of Game Animals Populations in Northern Europe. Petrozavodsk. P.17-18.

Bublichenko A.G. 2007. [Mammals] // Zvelev N.N. (ed.). [Natural Environment and Biodiversity of Berezoviye Islands Archipelago (the Gulf of Finland)]. Sankt-Peterburg: Boston-Spektr. P.311-312 [in Russian].

Bublichenko A.G., Bublichenko J.N., Volkova E.A., Isachenko G.A., Kataeva O.A., Kurbatova L.E., Potapov R.L., Galtsova V.V., Dmitriev V.V., Doronina A.Y., Ivanov V.V., Kulangieva L.V., Lange E.K., Lukjanov S.V., Pnjushkov A.V., Reznikov A.I., Stepanov O.V., Shilin M.B. \& Khramtsov V.N. 2003. [Natural Environment of the Coast and Water Area of the Gulf of Finland (Primorsk port Region)]. Sankt-Peterburg: Boston-Spektr. 128 pp. [in Russian]

Bublichenko A.G. \& Bublichenko J.N. 2005a. [Terrestrial vertebrate fauna] // Volkova E.A., Isachenko G.A. \& Khramtsov V.N. (eds). [Strelninsky Coast]. Sankt-Peterburg: Boston-Spektr. P.40-49 [in Russian].

Bublichenko A.G. \& Bublichenko J.N. 2005b. [Preliminary data on the terrestrial vertebrate fauna of "Gladyshevsky" complex nature reserve (Kurortny district, Saint-Petersburg)] // [Problems and Development Perspectives of Preserved Nature Areas of Saint-Petersburg]. Sankt-Peterburg. P.41-47 [in Russian].

Chistyakov D.V. 2002a. [Chiropterans (Chiroptera, Vespertilionidae) of the southern coast of the Gulf of Finland] // Trudy Biologicheskogo Nauchno-Issledovatel'skogo Instituta. T.48. P.164-171 [in Russian].

Chistyakov D.V. 2002b. [Wintering places of chiropterans in the underground (fortification structures mostly) constructions of Leningrad Region] // Trudy Biologicheskogo Nauchno-Issledovatel'skogo Instituta. T.48. P.172174 [in Russian].

Danilov P.I., Rusakov O.S. \& Tumanov I.L. 1979. [Carnivores of the North-Western part of USSR]. Leningrad: Nauka. 190 pp. [in Russian]

Hallanaro E.-L. \& Pylvänäjnen M. 2002. Nature in Northern Europe - Biodiversity in a Changing Environment. Copenhagen: NORD. 351 pp.

Ivanter E.V. 1986. [Animal World of Karelia. Mammals]. Petrozavodsk: Karelia. 224 pp. [in Russian]

Karaseva E.V. \& Telitsina A.Yu. 1996. [Methods of Rodents Investigation in Field Conditions]. Moskva: Nauka. 227 pp. [in Russian]

Köppen Fr.Th. 1883. Die Verbreitung des Elenthiers im Europäischen Russland, mit besonderer Berücksichtigung einer in den Fünfziger Jahren begonnenen Massenwanderung desselben // Beiträge zur Kenntniss des Russishen Reiches. Bd.2. S.141-240.

Martynov E.N. 1979. [Types of animal's habitat] // Lesovodstvo, Lesnye Kul'tury i Pochvovedenie. No.8. P.132136 [in Russian].

[Methodical Instructions for Game Animals Counting in the Forests of Russian Federation]. Moskva: ROSGIPROLES. 1997. 59 pp. [in Russian.]

[Methodical Directions for Organization, Carrying and Data Handling of Game Animals Winter Counting in Russian Federation]. Moskva: Glavohota. 1990. 48 pp. [in Russian]

Noskov G.A. (ed.). 2004. [Preserved nature of Karelian Isthmus]. Sankt-Peterburg: Professional. 312 pp. [in Russian]

Novikov G.A. 1953. [Field Investigations of Terrestrial Vertebrates Ecology]. Leningrad: Sovetskaya Nauka. 502 pp. [in Russian]

Novikov G.A., Airapetyants A.E., Pukinsky Yu.B., Strelkov P.P. \& Timofeeva E.K. 1970. [Mammals of Leningrad Region]. Leningrad: Izdatel'stvo Leningradskogo Gosudarstvennogo Universiteta. 359 pp. [in Russian]

Siivonen L. 1976. [Mammals of Northern Europe]. Moskva: Izdatel'stvo Lesnaya Promyshlennost. 232 pp. [in Russian]

Shnitnikov V.N. 1927. [Some data about mammal distribution in Leningrad Region] // Trudy Leningradskogo Obshchestva Estestvoispytatelei. T.57. No.1. P.59-72 [in Russian].

Timofeeva E.K. 1974. [Elk]. Leningrad: Izdatel'stvo Leningradskogo Gosudarstvennogo Universiteta. 167 pp. [in Russian]

Vereshchagin N.K. \& Rusakov O.S. 1979. [Ungulates of the North-Western part of USSR]. Leningrad: Nauka. 308 pp. [in Russian]

Volkova E.A., Isachenko G.A., Khramtsov V.N., Bublichenko A.G., Bublichenko J.N. \& Makarova M.A. 2001. [The complex Mapping of Natural Environment of the Coast of the Gulf of Finland (Luzskaya Bay Region)]. SanktPeterburg: Izdatel'stvo Khimicheskoi i Farmakologicheskoi Akademii. 140 pp. [in Russian, with English summary] 